\title{
Adverse effects of total hip arthroplasty on the hip abductor and adductor muscle lengths and moment arms during gait
}

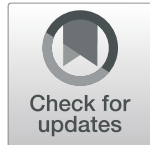

Xiangjun $\mathrm{Hu}^{1,2+}$, Nan Zheng ${ }^{1,2+}$, Wei-Chun Hsu ${ }^{3}$, Jingwei Zhang ${ }^{2}$, Huiwu Li ${ }^{2}$, Yunsu Chen ${ }^{4}$, Kerong Dai ${ }^{1,2}$ and Tsung-Yuan Tsai ${ }^{1,2^{*}}$ (1)

\begin{abstract}
Background: Precise evaluation of the hip abductor and adductor muscles function in total hip arthroplasty (THA) patients during gait could help prevent postoperative complications and optimize the rehabilitation training program. The purpose of this study was to elucidate the effects of THA on the hip abductor and adductor muscle lengths and moment arms of in vivo patients during gait.

Methods: Ten unilateral THA patients received CT scans and dual fluoroscopic imaging for the hip kinematics during gait. The hip abductor and adductor muscle insertions were digitized on the 3D hip model for the determination of their dynamic lines of action and moment arms. Changes in the hip abductor and adductor muscle lengths and moment arms of THA patients between the implanted and non-implanted sides were quantified during gait.
\end{abstract}

Results: The adductor longus, adductor brevis, and pectineus of the implanted hips had significantly $(P<0.05)$ less elongation than that of the non-implanted side during the stance phase. The gluteus medius, gluteus minimus, and piriformis moment arms of the implanted side were significantly shorter. The piriformis muscle moment arm was significantly larger. In the double support phase, the adductor magnus and adductor longus moment arms of the implanted sides were significantly decreased.

Conclusions: Results suggested that the adverse effects of THA on hip stability. Development of a rehabilitation program considering the effects of THA is essential. Accurate surgical techniques may reduce the impact of THA on the peripheral muscles.

Keywords: Total hip arthroplasty, Biomechanics, Muscle length, Moment arm, Hip abductor muscle, Hip adductor muscle

\footnotetext{
* Correspondence: tytsai@sjtu.edu.cn

'Xiangjun Hu and Nan Zheng contributed equally to this work.

'School of Biomedical Engineering \& Med-X Research Institute, Shanghai Jiao

Tong University; Engineering Research Center of Digital Medicine and Clinical

Translation, Ministry of Education, Shanghai, China

${ }^{2}$ Shanghai Key Laboratory of Orthopaedic Implants \& Clinical Translation R\&D

Center of 3D Printing Technology, Department of Orthopaedic Surgery,

Shanghai Ninth People's Hospital, Shanghai Jiao Tong University School of

Medicine, Shanghai, China

Full list of author information is available at the end of the article
}

C C The Author(s). 2020 Open Access This article is licensed under a Creative Commons Attribution 4.0 International License, which permits use, sharing, adaptation, distribution and reproduction in any medium or format, as long as you give appropriate credit to the original author(s) and the source, provide a link to the Creative Commons licence, and indicate if changes were made. The images or other third party material in this article are included in the article's Creative Commons licence, unless indicated otherwise in a credit line to the material. If material is not included in the article's Creative Commons licence and your intended use is not permitted by statutory regulation or exceeds the permitted use, you will need to obtain permission directly from the copyright holder. To view a copy of this licence, visit http://creativecommons.org/licenses/by/4.0/ The Creative Commons Public Domain Dedication waiver (http://creativecommons.org/publicdomain/zero/1.0/) applies to the data made available in this article, unless otherwise stated in a credit line to the data. 


\section{Background}

Total hip arthroplasty (THA) is a standard surgical procedure for the treatment of end-stage hip osteoarthritis and avascular necrosis of the femoral head. THA can effectively relieve pain, restore hip function, and improve the quality of life [1]. However, asymmetric gait, pelvic drop, and limping were reported in patients after THA [2]. These dysfunctions can lead to continuous soft tissue contraction around the hip joint, resulting in prosthesis dislocation and loosening, periprosthetic fracture, and unequal lower limb length [3].

Previous studies reported that the hip muscle function could be affected after THA. Demos et al. [4] found that although THA can improve walking function in most patients, $11.6 \%$ of patients still suffered from moderate to severe claudication or limping. During the single-leg support period, the trunk swings to the operated leg, and the limp is swaying left and right on the coronal plane due to the lack of hip abductor and adductor muscle strength [5]. Moreover, studies have demonstrated that insufficient muscle strength and tension in the hip abductor and adductor muscles (nerve injury or limb shortening) are the causes of THA dislocation [6, 7]. Rudiger et al. [8] reported that the decrease in the femoral offset after THA would reduce the abductor moment arms during gait using the musculoskeletal model in OpenSim. Precise evaluation of the function of the hip abductor and adductor muscles in THA patients during gait could help prevent postoperative complications and optimize the rehabilitation training program. However, precise biomechanical effects of THA on the hip abductor and adductor muscles of in vivo patients during gait remains unknown.

The dual fluoroscopic imaging system (DFIS)-based tracking technique has been extensively applied to capture the motion of in vivo lower limb joints during gait and allowed accurate quantification of 3D joint kinematics [9-11]. Recently, some researchers have used the DFIS for the measurement of soft tissue length changes during motion [12, 13]. However, it has not been used to quantify the effects of THA on the hip muscle lengths and moment arms. This study aimed (1) to quantify the hip abductor and adductor muscle lengths and moment arms in unilateral THA patients during gait and (2) to analyze the effects of THA on the biomechanical parameters of the hip abductor and adductor muscles.

\section{Materials and methods}

\section{Subject recruitment and tasks}

Our Institutional Review Board approved this study. Ten THA patients ( 8 women, 2 men; age $61.9 \pm 9.4$ years) with no history of any surgical and orthodontic treatment were recruited. All the patients were with end-stage hip osteoarthritis before THA. The average follow-up time was
1 year (10.2 \pm 1.6 months). Harris hip scores (HHS) of all the patients were higher than 90. These patients received unilateral THA from the same surgeon using the posterior approach. All subjects received a computed tomography (CT) scan (SOMATOM Definition AS1, Siemens), providing an in-plane resolution of $0.98 \mathrm{~mm}$ at a $1-\mathrm{mm}$ slice thickness, from the L5 vertebra to the tibial plateau for the construction of surface models of the femur, acetabular cup, pelvis, and prosthesis. The patients performed treadmill walking at self-selected speed under the DFIS surveillance (BV Pulsera, Phillips Medical, USA) for both the implanted and non-implanted hips.

\section{The 3D skeletal model reconstruction and hip kinematics measurement during gait}

We segmented the CT images and reconstructed the 3D surface models of the femoral stem, acetabular cup, pelvis, and prosthesis of both sides using a region growing method (Amira, Thermo Fisher Scientific, Waltham, Massachusetts, USA). The reconstructed 3D surface models were imported into MATLAB (R2018a, MathWorks, Natick, MA, USA) to establish anatomical coordinate systems (ACS) for the pelvis and femur following the recommendation of the International Society of Biomechanics [14].

The DFIS projection geometry was obtained through the system calibration procedure for the creation of a virtual environment in software [15]. The six degrees of freedom (6-DoF) of the hip can be determined by matching the contours of the 3D models, and their fluoroscopic projections [9]. The measurement errors were $0.35 \mathrm{~mm}$ and $0.55^{\circ}$ in measuring the hip joint translations and rotations, respectively [9].

\section{Muscle lengths and moment arms quantification}

The 3D skeleton models were imported into MATLAB for the attachment area determination of the hip abductor and adductor muscles in the pelvis and femur of the native side. The muscle attachment sites were digitized following human anatomy studies [16]. We stratified the hip muscles into two groups according to their functions. First, the abductor muscle group includes the gluteus medius (GMD), gluteus minimus (GMI), and piriformis (PF). Second, the adductor muscle group includes the adductor magnus (AM), adductor longus $(\mathrm{AL})$, adductor brevis $(\mathrm{AB})$, and pectineus $(\mathrm{PT})$. If the longitudinal direction of the muscle bundles was straight, the center of the attachment was determined as the starting and ending points to simulate each muscle's lines of action [17]. If the longitudinal direction of the muscle bundles was curve, the bone structure turning point was taken as the ending point [16]. If the muscle attachment area was wide, we divided it into bundles to 
reflect the function of different muscle bundles (Fig. 1). To determine the muscle attachments of the implanted side, we mirrored and aligned the non-implanted femur and muscle attachments to the remaining femur of the implanted side [10] (Fig. 1).

The dynamic changes of each muscle length and moment arm during gait were calculated using in-vivo 6DoF hip joint kinematics. The center of the femoral head was considered as the point of action for the lower limb muscles. Moment arm was defined as the distance from the center of the femoral head to each muscle's lines of action. The in vivo hip muscle lengths and moment arms data of both the implanted and non-implanted hips against the gait cycle were calculated and compared.

\section{Statistical analysis}

The Wilcoxon signed-rank test was used to determine whether there was a significant difference in hip muscle lengths and moment arms between the implanted and non-implanted sides during gait. A level of significance was set at 0.05 . All the statistics were performed in MATLAB. Statistical power analyses were computed in G*power (Franz Faul, Christian-Albrechts-Universität Kiel, Germany).

\section{Results}

The difference in muscle lengths

The adductor muscles of the implanted side were significantly $(P<0.05)$ shorter than that of the contralateral side (Fig. 2j-l) with maximum differences of $2.9 \mathrm{~mm}, 2.9$ $\mathrm{mm}$, and $3.6 \mathrm{~mm}$ for the $\mathrm{AL}, \mathrm{AB}$, and $\mathrm{PT}$, respectively (Table 1). No significant differences were found in the abductor muscle lengths between the implanted and non-implanted sides during gait (Fig. 2).

\section{The difference in muscle moment arms}

For the abductor muscles in the stance phase during gait, the GMDA, GMIM, and GMIP moment arms of the implanted side were significantly shorter than those of the contralateral side $(P<0.05)$ with the maximum differences of $1.7 \mathrm{~mm}, 1.5 \mathrm{~mm}$, and $2.5 \mathrm{~mm}$, respectively (Fig. 3a, e-f, Table 2). The GMDM and GMDP of the implanted hips showed significantly shorter moment arms-not only in the stance phase but also in the partial swing phase (Fig. 3b-c) with maximum differences of $2.5 \mathrm{~mm}$ and $3.0 \mathrm{~mm}$ (Table 2). For the adductor muscle in the double support phase during gait, the AMP (Fig. 3i) and AL (Fig. 3j) moment arms of the implanted sides were significantly shorter than those of the non-implanted sides. Moreover, in the terminal swing phase, a significantly

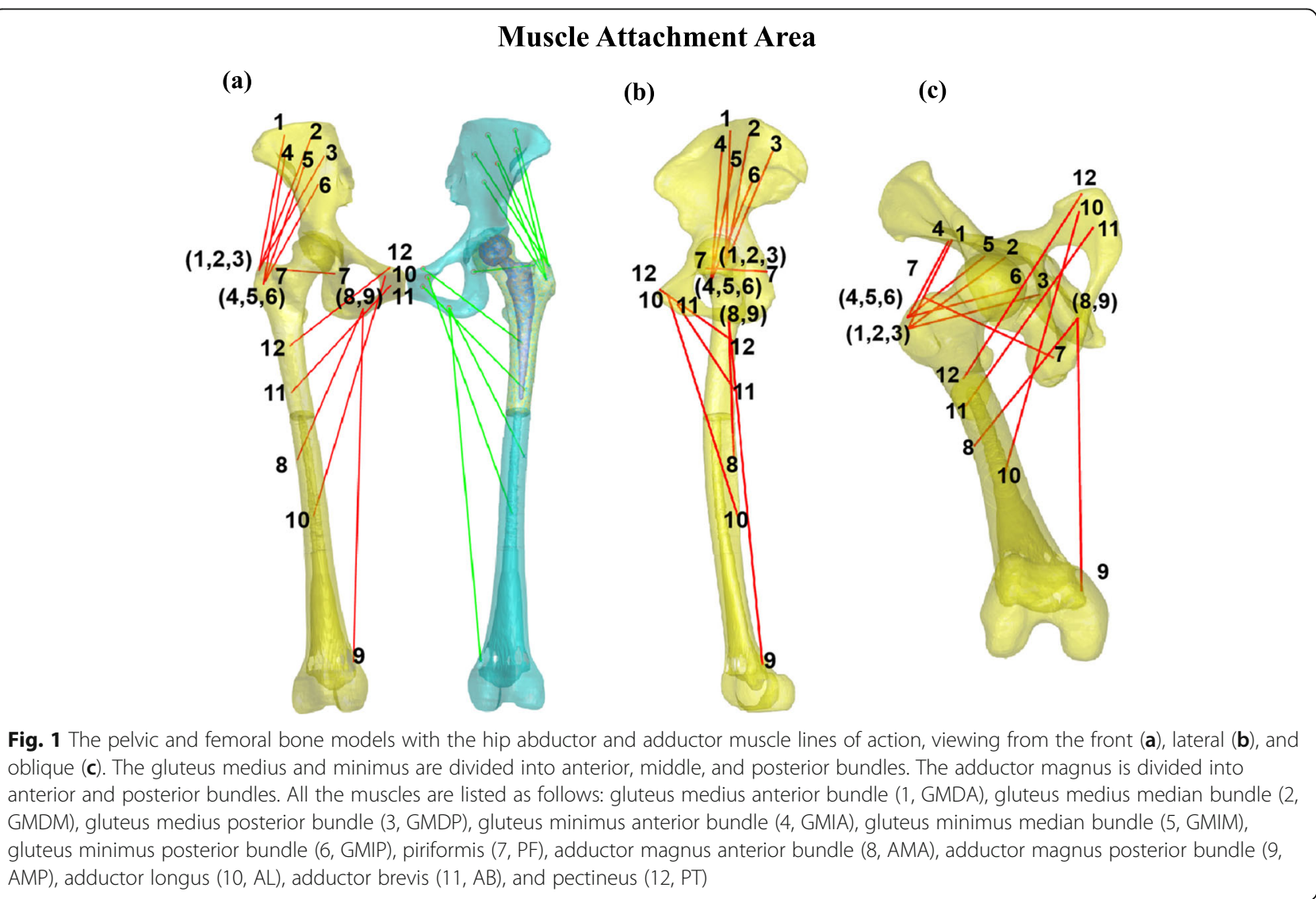



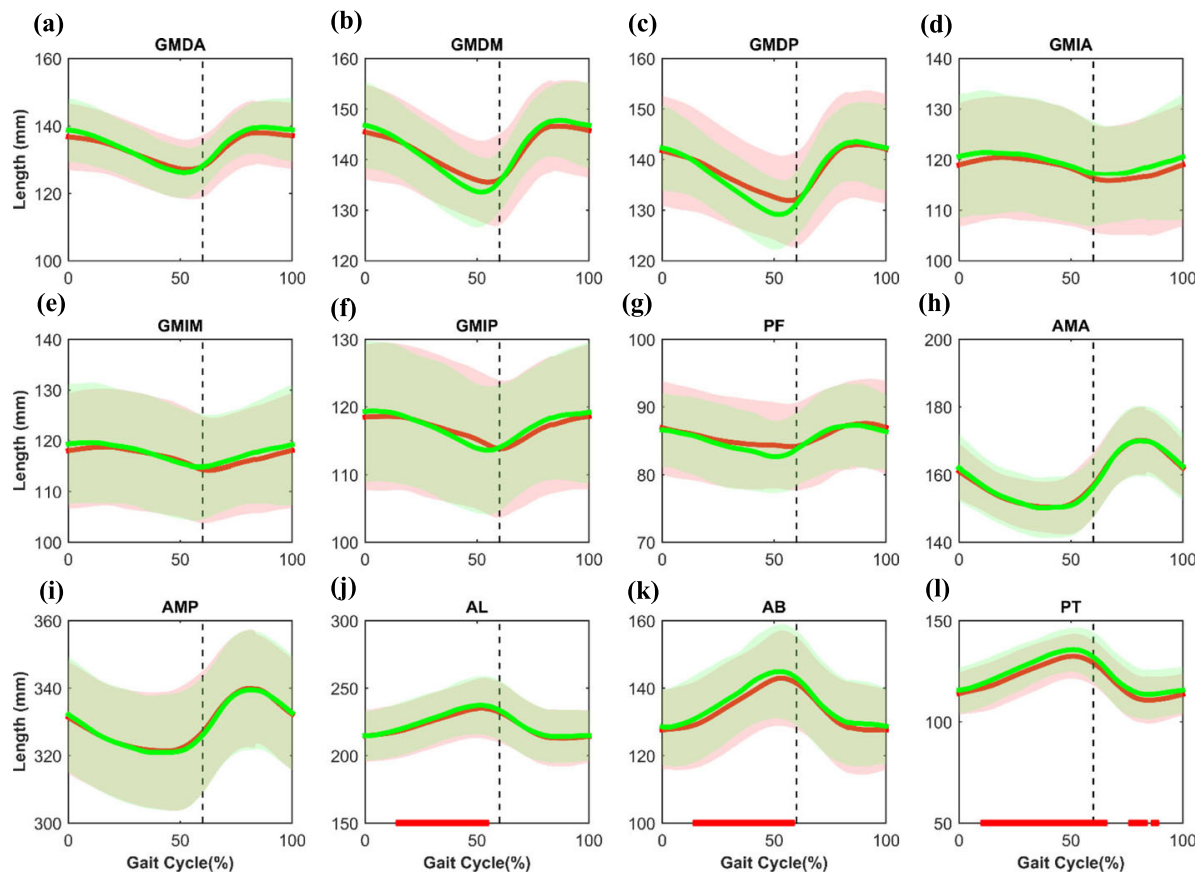

Fig. 2 The average and standard deviation of the hip muscle lengths in THA and contralateral non-operated hip are illustrated during gait. The black vertical dashed lines indicate toe-off. The red and green lines represent the implanted and non-implanted sides. Statistical significance between the legs is marked by the bold red line along the $X$-axis of each graph. Muscle abbreviations are in Fig. 1 caption

shorter moment arm $(P<0.05)$ (Fig. 3j) was observed in the AL of the implanted side. The maximum differences were $1.5 \mathrm{~mm}$ and $1.2 \mathrm{~mm}$ (Table 2). The PT moment arm (Fig. 3l) was significantly larger $(P<0.05)$ than that of the non-operated side almost during the entire gait, with maximum difference of $2.2 \mathrm{~mm}$ (Table 2).

\section{Discussion}

Statistically significant differences were found in the hip abductor and adductor muscle lengths and moment arms between the legs in unilateral THA patients during gait. Significantly shorter abductor moment arms, less elongated adductor muscle, and longer adductor muscle

Table 1 Maximum shortening and elongation in each adductor and abductor muscle of the implanted side relative to the nonimplanted side are listed throughout gait

\begin{tabular}{|c|c|c|c|c|c|c|c|c|c|c|c|c|}
\hline \multirow[t]{3}{*}{ Muscle length } & \multicolumn{7}{|c|}{ Abductor muscles } & \multicolumn{5}{|c|}{ Adductor muscles } \\
\hline & \multicolumn{3}{|l|}{ GMD } & \multicolumn{3}{|l|}{ GMI } & \multirow[t]{2}{*}{ PF } & \multicolumn{2}{|l|}{$\overline{A M}$} & \multirow[t]{2}{*}{$\mathrm{AL}$} & \multirow[t]{2}{*}{$A B$} & \multirow[t]{2}{*}{ PT } \\
\hline & GMDA & GMDM & GMDP & GMIA & GMIM & GMIP & & AMA & AMP & & & \\
\hline \multicolumn{13}{|c|}{ Maximum shortening } \\
\hline Mean (mm) & -2.0 & -1.3 & -0.9 & -1.9 & -1.6 & -1.3 & -0.6 & -0.9 & -0.8 & -2.9 & -2.9 & -3.6 \\
\hline $\mathrm{SD}(\mathrm{mm})$ & 5.2 & 6.1 & 6.5 & 4.3 & 4.5 & 4.8 & 3.6 & 3.6 & 3.5 & 3.7 & 3.5 & 4.0 \\
\hline$P$ value & 0.215 & 0.648 & 0.648 & 0.085 & 0.372 & 0.617 & 0.913 & 0.286 & 0.133 & $0.014^{*}$ & $0.008^{*}$ & $0.007^{*}$ \\
\hline \multicolumn{13}{|c|}{ Maximum elongation } \\
\hline Mean (mm) & 1.1 & 2.3 & 3.4 & N.A. & 0.5 & 1.4 & 1.7 & 0.6 & 1.0 & 0.2 & N.A. & N.A. \\
\hline $\mathrm{SD}(\mathrm{mm})$ & 4.8 & 5.3 & 6.0 & N.A. & 4.5 & 4.9 & 4.3 & 5.1 & 5.0 & 5.0 & N.A. & N.A. \\
\hline$P$ value & 0.420 & 0.170 & 0.078 & N.A. & 0.586 & 0.349 & 0.133 & 0.811 & 0.586 & 0.617 & N.A. & N.A. \\
\hline
\end{tabular}

Muscle abbreviations are in Fig. 1 caption

A Wilcoxon signed-rank test was used to determine whether there is a significant difference

N.A. not available

${ }^{*} P$ value $<0.05$ 


\section{Muscle moment Arms}
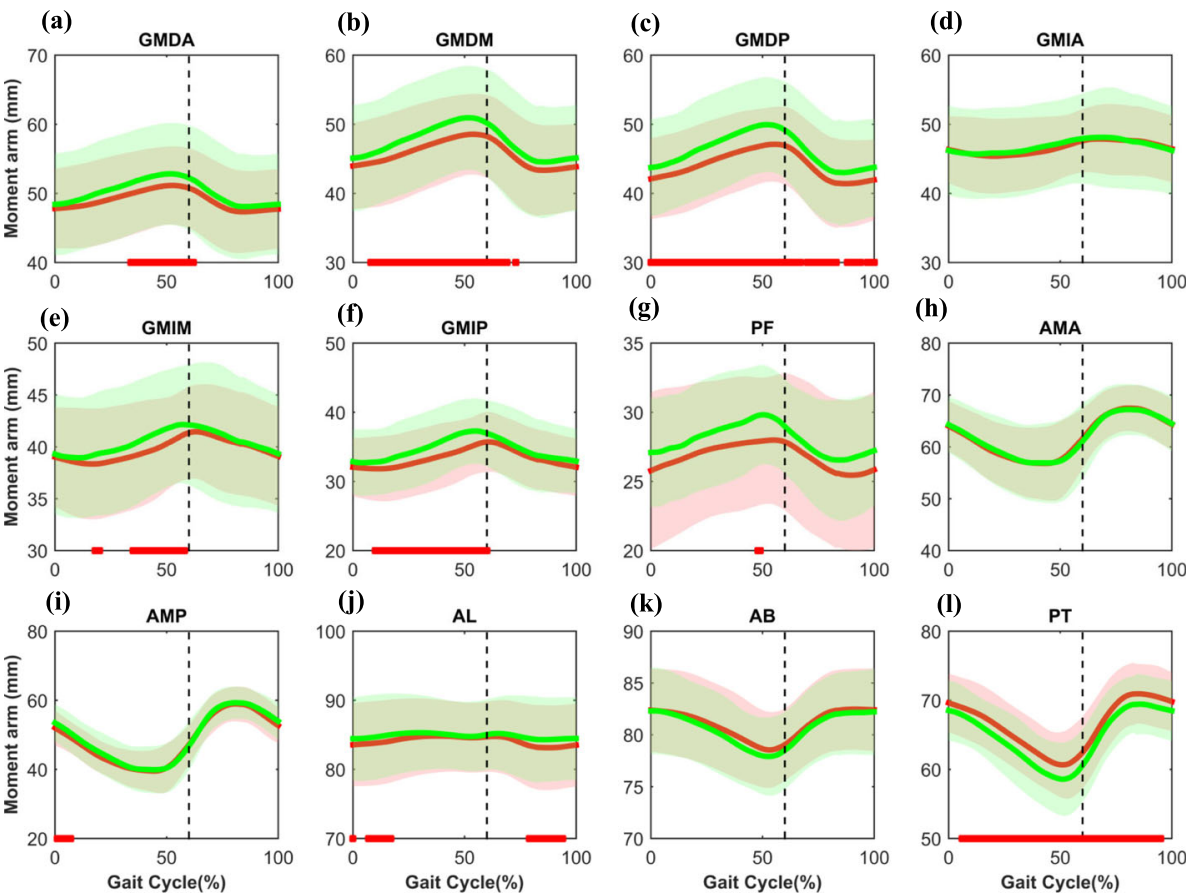

Fig. 3 The average and standard deviation of the hip muscle moment arms in THA and contralateral non-operated hip are illustrated during gait. The black vertical dashed lines indicate the end of the stance phase. The red and green lines represent the implanted and non-implanted sides. Statistical significance between the legs is marked by the bold red line along the $X$-axis of each graph. Muscle abbreviations are in Fig. 1 caption

moment arms during the stance phase of the gait cycle were observed in the implanted side than the contralateral non-implanted side $(P<0.05$, Tables 1 and 2 , Figs. 2 and 3). Among all the hip abductor and adductor muscles, the GMDP and PT moment arms were affected the most with the highest difference during most of the gait cycle (Figs. 2 and 3). The GMDP, AMP, and AL moment arms significantly decreased during the load-bearing response period (Fig. 3). Our findings indicated that THA would change the biomechanical parameters of the hip

Table 2 Maximum shortening and elongation of muscle moment arm in each adductor and abductor muscle of the implanted side relative to the non-implanted side are listed throughout gait

\begin{tabular}{|c|c|c|c|c|c|c|c|c|c|c|c|c|}
\hline \multirow{3}{*}{$\begin{array}{l}\text { Muscle } \\
\text { moment arm }\end{array}$} & \multicolumn{7}{|c|}{ Abductor muscles } & \multicolumn{5}{|c|}{ Adductor muscles } \\
\hline & \multicolumn{3}{|l|}{$\overline{G M D}$} & \multicolumn{3}{|l|}{ GMI } & \multirow[t]{2}{*}{ PF } & \multicolumn{2}{|l|}{$\overline{A M}$} & \multirow[t]{2}{*}{$\mathrm{AL}$} & \multirow[t]{2}{*}{$A B$} & \multirow[t]{2}{*}{ PT } \\
\hline & GMDA & GMDM & GMDP & $\overline{G M I A}$ & GMIM & GMIP & & $\overline{A M A}$ & AMP & & & \\
\hline \multicolumn{13}{|c|}{ Maximum shortening } \\
\hline Mean (mm) & -1.7 & -2.5 & -3.0 & -0.7 & -1.5 & -2.5 & -1.9 & -0.5 & -1.5 & -1.2 & N.A. & N.A. \\
\hline $\mathrm{SD}(\mathrm{mm})$ & 3.4 & 3.2 & 3.2 & 2.8 & 2.4 & 2.4 & 3.4 & 2.1 & 2.3 & 1.9 & N.A. & N.A. \\
\hline$P$ value & $0.025^{*}$ & $0.010^{*}$ & $0.008^{*}$ & 0.122 & $0.022^{*}$ & $0.008^{*}$ & 0.053 & 0.472 & $0.048^{*}$ & $0.039^{*}$ & N.A. & N.A. \\
\hline \multicolumn{13}{|c|}{ Maximum elongation } \\
\hline Mean (mm) & N.A. & N.A. & N.A. & 0.1 & N.A. & N.A. & N.A. & 0.6 & 0.5 & N.A. & 0.8 & 2.2 \\
\hline $\mathrm{SD}(\mathrm{mm})$ & N.A. & N.A. & N.A. & 3.1 & N.A. & N.A. & N.A. & 2.9 & 4.9 & N.A. & 1.6 & 2.1 \\
\hline$P$ value & N.A. & N.A. & N.A. & 0.811 & N.A. & N.A. & N.A. & 0.306 & 0.811 & N.A. & 0.071 & $0.005^{*}$ \\
\hline
\end{tabular}

Muscle abbreviations are in Fig. 1 caption

A Wilcoxon signed-rank test was used to determine whether there is a significant difference

N.A. not available

${ }^{*} P$ value $<0.05$ 
abductor and adductor muscles, which could result in muscle weakness and limit the effectiveness of regular rehabilitation.

The muscle lengths and moment arms are essential biomechanical parameters for muscle function evaluation. Dostal et al. [17] quantified hip muscle lengths and moment arms in a male cadaver by dissecting the proximal and distal muscle attachments. They reported that the GMD, GMI, and AM anterior bundles provided a relatively larger moment arm in each muscle. A clinical commentary study reviewed the hip muscle function based on a hypothetical model and reported that GMD has the largest abduction moment arm in the abductor muscles [18]. Our results are in line with the abovementioned findings. Further, Bjørdal et al. [19] reported a larger abductor moment arm in the implanted side (65.4 $\pm 5.9 \mathrm{~mm})$ than in the non-implanted side $(58.0 \pm 6.6$ $\mathrm{mm}$ ) on radiographs of 148 THA patients. In contrast, we found shorter abductor moment arms after THA. This difference may attribute to different measurement methods, surgical approaches, and implant systems used between studies.

Abductor deficiency has been reported after THA $[2,20]$. Previous studies demonstrated that when the hip abductor is weak, the pelvis drops to the sound side, leading to gait instability $[3,7]$. In this study, we found that the abductor muscle lengths of the THA side were longer than the contralateral non-implanted side during the support phase (Fig. 2), implying less abductor muscle contraction in the THA side. Furthermore, the moment arms of these abductor muscles were smaller than that of the contralateral sound side (Fig. 3), resulting in reduced abductor moment and muscle efficiency. On the other hand, previous studies reported that many THA patients experienced persistent adductor muscle contracture [21], which collaborates with the significant adductor muscle shortening than that of the non-implanted side in our study (Fig. 2). The abductor muscle weakness and contracture observed in THA patients may relate to the adverse biomechanical effects of THA.

The adductor and abductor muscles also assist hip flexion, extension, and rotation. The AM began to pull at the end of the loading response period. The AM and GMDP help the gluteus maximus muscle in hip extension, according to the previous studies [18]. In addition to the AM and GMDP, AL can also act as a hip flexor and extensor [18]. In this study, we found that AMP, GMDP, and $\mathrm{AL}$ of the THA side were shortened during the loading response period, implying concentrically contraction. Additionally, their moment arms are significantly smaller than the non-operated side (Figs. 2 and 3). The GMIP was considered as a secondary external rotator of the hip [18]. In this study, we observed that the GMIP of the THA side had a significantly shorter moment arm, which might associate with the excessive internal rotation reported in THA patients [10]. The adverse biomechanical effects may associate with the hip extensor weakness, lower hip range of motion, and abnormal hip internal rotation during gait (Figs. 2 and 3).

In this study, Harris hip scores (HHS) of all the patients were higher than 90, indicating these THA patients were well-functioning. However, significant changes in hip abductor and adductor muscle lengths and moment arms in the implanted side were observed. In clinical practice, it is challenging to identify the malfunction of each muscle non-invasively. Our study provided a new quantitative method to evaluate the in vivo performance of each muscle during functional activity, which was essential to develop a personalized rehabilitation strategy after surgery for better clinical outcomes.

The Sherrington's reciprocal inhibition principle states that tensioned or shortened antagonist muscles may reflexively inhibit active muscles [22]. The hip adductor muscle is the antagonist of the hip abductor muscle. If the hip adductor muscle becomes tight and shortened, it may, in turn, be a cause of hip abductor muscle weakness. Our study found that in THA patients, the hip adductors of the operated side were shortened compared with the non-operated side during gait, which may reflexively inhibit the hip abductor muscle. The current rehabilitation training for patients after THA focuses more on strengthening the hip abductor muscles, which may be limited. The previous studies reported asymmetric muscle activities even 2 years after THA [23, 24]. Therefore, the training of antagonist muscles should be enhanced together. We suggest that THA patients should first start to recover the hip adductor muscle tension and length, especially the PT, the most affected adductor, through muscle energy technique [25] and specific fascial release technique. Subsequently, strengthen the weak or inhibited abductor hip muscles, especially the GMD, the most affected abductor.

Janda [26] reported that tensed or shortened antagonist muscles often become active in unrelated movements, further worsening dynamic posture control. This compensation will naturally decrease hip extension and increase hip internal rotation and adduction [10]. In this study, the decreased GMDP, AMP, and AL moment arms after THA may decrease hip extension, and GMIP may increase hip internal rotation during the gait cycle. This compensatory model will lead to the biomechanical changes of the entire lower limb, which in turn will lead to incorrect movements and aggravates injuries [27, 28]. It is suggested that postural control, combined with individualized muscle training, should be implemented in patients after THA to maintain the body's overall balance by strengthening the coordination and control ability between the muscle groups. 
Biomechanical changes in the musculoskeletal system after THA are closely related to surgical techniques and prosthesis design [8]. Accurate surgical techniques as well as optimal prosthesis design and positioning, can reduce the impact of joint replacement on peripheral muscle function. A previous study suggested that accurate intraoperative femoral offset reconstruction is essential for maintaining the abductor muscle moment arm [8]. The displacement of the center of the femoral head may occur due to surgeon preferences (e.g., excessive correction of native femoral anteversion), inaccurate preoperative templating, or limited selection of available implants. Currently, the hip prosthesis design features a smaller femoral head, higher rotation center, wider neck, and lower range of motion, which negatively affects the biomechanical parameters and peripheral muscle performance [29]. We recommend that the next generation hip prosthesis geometry should be optimized for better functional recovery and clinical outcomes.

The present study should be interpreted in light of potential limitations. Only 10 patients who received THA for a year participated. However, using the muscle lengths difference between the implanted and nonimplanted sides, the statistical verification power of the data is $97 \%$ through G-Power calculation. Future studies should recruit more patients who underwent THA with preoperative data and longer follow-up to compare the effects of component positioning, follow-up times, and rehabilitation program on muscle function recovery.

\section{Conclusion}

This study quantified the in vivo dynamic hip abductor and adductor muscle lengths and moment arms in unilateral THA patients during gait using the DFIS tracking technique. Significantly shorter abductor moment arm, less elongated adductor muscle, and longer adductor muscle moment arm during the stance phase of the gait cycle were observed in the implanted side than the contralateral non-implanted side. We suggest that patients after THA should first recover the normative length of hip adductor muscles, which can be achieved through physiological treatment of muscle energy technique. Moreover, postural control combined with individualized muscle training is recommended in patients after THA to maintain the overall balance of the body by strengthening the coordination and control ability between the abductor and adductor muscle groups. Accurate surgical techniques, as well as optimal prosthesis design and positioning, could reduce the impact of joint replacement on the biomechanical parameters of the peripheral muscles. Further studies are guaranteed to minimize the adverse effects of THA on the surrounding muscles.

\section{Abbreviations}

THA: Total hip arthroplasty; DFIS: Dual fluoroscopic imaging system; CT: Computed tomography; 6-DoF: Six degrees of freedom; GMD: Gluteus medius; GMDA: Gluteus medius anterior bundle; GMDM: Gluteus medius median bundle; GMDP: Gluteus medius posterior bundle; GMl: Gluteus minimus; GMIA: Gluteus minimus anterior bundle; GMIM: Gluteus minimus median bundle; GMIP: Gluteus minimus posterior bundle; PF: Piriformis; AM: Adductor magnus; AMA: Adductor magnus anterior bundle; AMP: Adductor magnus posterior bundle; AL: Adductor longus; AB: Adductor brevis; PT: Pectineus

\section{Acknowledgements \\ Not applicable}

\section{Authors' contributions}

$\mathrm{XJH}, \mathrm{NZ}, \mathrm{YSC}$, and TYT contributed to the conception and design. XJH, NZ, YSC, JWZ, HWL, and TYT contributed to the provision of study materials or patients and collection and assembly of data. XJH and TYT carried out the data analysis. XJH, NZ, WCH, JWZ, HWL, KRD, and TYT contributed to data interpretation. $\mathrm{XJH}, \mathrm{NZ}$, and TYT participated in the writing of the manuscript. All authors read and approved the final manuscript.

\section{Funding}

This project was sponsored by the National Natural Science Foundation of China (31771017, 31972924, 31900941), the Science and Technology Commission of Shanghai Municipality (16441908700), the Innovation Research Plan supported by Shanghai Municipal Education Commission (ZXWF082101), the National Key R\&D Program of China (2017YFC0110700, 2019YF(0120600), and the Interdisciplinary Program of Shanghai Jiao Tong University (ZH2018QNA06, YG2017MS09).

\section{Availability of data and materials}

The datasets used and analyzed during the current study are available from the corresponding author on reasonable request.

\section{Ethics approval and consent to participate}

The protocol was approved by the Ethics Committee of Shanghai Sixth People's Hospital, China (No. 2019026). Written informed consent was obtained from all patients enrolled in the investigation.

Consent for publication

Not applicable

\section{Competing interests}

The authors declare that they have no competing interests.

\section{Author details}

${ }^{1}$ School of Biomedical Engineering \& Med-X Research Institute, Shanghai Jiao Tong University; Engineering Research Center of Digital Medicine and Clinical Translation, Ministry of Education, Shanghai, China. ${ }^{2}$ Shanghai Key Laboratory of Orthopaedic Implants \& Clinical Translation R\&D Center of 3D Printing Technology, Department of Orthopaedic Surgery, Shanghai Ninth People's Hospital, Shanghai Jiao Tong University School of Medicine, Shanghai, China. ${ }^{3}$ Graduate Institute of Biomedical Engineering, National Taiwan University of Science and Technology, Taipei, Taiwan. ${ }^{4}$ Department of Orthopaedic, Shanghai Jiao Tong University Affiliated Sixth People's Hospital, Shanghai, China.

Received: 22 June 2020 Accepted: 29 July 2020

Published online: 12 August 2020

\section{References}

1. Park KK, Tsai TY, Dimitriou D, Kwon YM. Three-dimensional in vivo difference between native acetabular version and acetabular component version influences iliopsoas impingement after total hip arthroplasty. Int Orthop. 2016;40(9):1807-12. https://doi.org/10.1007/s00264-015-3055-5.

2. Kolk S, Minten MJ, van Bon GE, Rijnen WH, Geurts AC, Verdonschot N, et al. Gait and gait-related activities of daily living after total hip arthroplasty: a systematic review. Clin Biomechanics (Bristol, Avon). 2014;29(6):705-18. https://doi.org/10.1016/j.clinbiomech.2014.05.008. 
3. Bahl JS, Nelson MJ, Taylor M, Solomon LB, Arnold JB, Thewlis D. Biomechanical changes and recovery of gait function after total hip arthroplasty for osteoarthritis: a systematic review and meta-analysis. Osteoarthr Cartil. 2018; 26(7):847-63. https://doi.org/10.1016/j.joca.2018.02.897.

4. Demos HA, Rorabeck CH, Bourne RB, MacDonald SJ, McCalden RW. Instability in primary total hip arthroplasty with the direct lateral approach. Clin Orthop Relat Res. 2001;393:168-80. https://doi.org/10.1097/00003086200112000-00020.

5. Perron M, Malouin F, Moffet H, McFadyen BJ. Three-dimensional gait analysis in women with a total hip arthroplasty. Clin Biomechanics (Bristol, Avon). 2000;15(7):504-15. https://doi.org/10.1016/s0268-0033(00)00002-4

6. Alberton GM, High WA, Morrey BF (2002) Dislocation after revision total hip arthroplasty: an analysis of risk factors and treatment options. The Journal of bone and joint surgery American volume 84-a (10):1788-1792.

7. Pierchon F, Pasquier G, Cotten A, Fontaine C, Clarisse J, Duquennoy A. Causes of dislocation of total hip arthroplasty. CT study of component alignment. J Bone Joint Surg Br. 1994;76(1):45-8.

8. Rudiger HA, Guillemin M, Latypova A, Terrier A. Effect of changes of femoral offset on abductor and joint reaction forces in total hip arthroplasty. Arch Orthop Trauma Surg. 2017;137(11):1579-85. https://doi.org/10.1007/s00402017-2788-6.

9. Tsai TY, Li JS, Wang S, Lin H, Malchau H, Li G, et al. A novel dual fluoroscopic imaging method for determination of THA kinematics: in-vitro and in-vivo study. J Biomech. 2013;46(7):1300-4. https://doi.org/10.1016/j. jbiomech.2013.02.010.

10. Tsai TY, Dimitriou D, Li JS, Woo Nam K, Li G, Kwon YM. Asymmetric hip kinematics during gait in patients with unilateral total hip arthroplasty: in vivo 3-dimensional motion analysis. J Biomech. 2015;48(4):555-9. https://doi.org/10.1016/j.jbiomech.2015.01.021.

11. Tsai TY, Li JS, Dimitriou D, Kwon YM. Does component alignment affect gait symmetry in unilateral total hip arthroplasty patients? Clin Biomechanics (Bristol, Avon). 2015;30(8):802-7. https://doi.org/10.1016/j.clinbiomech.2015. 06.010 .

12. Kernkamp WA, Wang C, Li C, Hu H, van Arkel ERA, Nelissen $\mathrm{R}$, et al. The medial patellofemoral ligament is a dynamic and anisometric structure: an in vivo study on length changes and isometry. Am J Sports Med. 2019:47(7): 1645-53. https://doi.org/10.1177/0363546519840278.

13. Kernkamp WA, Jens AJT, Varady NH, van Arkel ERA, Nelissen R, Asnis PD, et al. Anatomic is better than isometric posterior cruciate ligament tunnel placement based upon in vivo simulation. Knee Surgery Sports Traumatol Arthroscopy. 2018. https://doi.org/10.1007/s00167-018-5233-7.

14. Wu G, Siegler S, Allard P, Kirtley C, Leardini A, Rosenbaum D, et al. ISB recommendation on definitions of joint coordinate system of various joints for the reporting of human joint motion--part l: ankle, hip, and spine. International Society of Biomechanics. J Biomech. 2002;35(4):543-8. https://doi.org/10.1016/s0021-9290(01)00222-6.

15. Bingham J, Li G. An optimized image matching method for determining invivo TKA kinematics with a dual-orthogonal fluoroscopic imaging system. J Biomech Eng. 2006;128(4):588-95. https://doi.org/10.1115/1.2205865.

16. Brand RA, Crowninshield RD, Wittstock CE, Pedersen DR, Clark CR, van Krieken FM. A model of lower extremity muscular anatomy. J Biomech Eng. 1982;104(4):304-10. https://doi.org/10.1115/1.3138363.

17. Dostal WF, Andrews JG. A three-dimensional biomechanical model of hip musculature. J Biomech. 1981;14(11):803-12. https://doi.org/10.1016/00219290(81)90036-1.

18. Neumann DA. Kinesiology of the hip: a focus on muscular actions. J Orthopaedic Sports Phys Therapy. 2010;40(2):82-94. https://doi.org/10.2519/ jospt.2010.3025.

19. Bjordal F, Bjorgul K. The role of femoral offset and abductor lever arm in total hip arthroplasty. J Orthop Traumatol. 2015;16(4):325-30. https://doi. org/10.1007/s10195-015-0358-7

20. Liu Q, Cheng X, Yan D, Zhou Y. Plain radiography findings to predict dislocation after total hip arthroplasty. J Orthop Translat. 2019;18:1-6. https://doi.org/10.1016/j.jot.2018.12.003.

21. Santamato A, Ranieri M, Panza F, Solfrizzi V, Frisardi V, Lapenna LM, et al. Botulinum toxin type a in the treatment of painful adductor muscle contracture after total hip arthroplasty. Orthopedics. 2009;32(10). https://doi. org/10.3928/01477447-20090818-29.

22. De Luca CJ, Mambrito B. Voluntary control of motor units in human antagonist muscles: coactivation and reciprocal activation. J Neurophysiol. 1987:58(3):525-42. https://doi.org/10.1152/jn.1987.58.3.525.
23. Jensen C, Aagaard P, Overgaard S. Recovery in mechanical muscle strength following resurfacing vs standard total hip arthroplasty - a randomised clinical trial. Osteoarthr Cartil. 2011;19(9):1108-16. https://doi.org/10.1016/j. joca.2011.06.011.

24. Kennedy DM, Stratford PW, Robarts S, Gollish JD. Using outcome measure results to facilitate clinical decisions the first year after total hip arthroplasty. J Orthopaedic Sports Phys Therapy. 2011;41(4):232-9. https://doi.org/10. 2519/jospt.2011.3516.

25. Goodridge JP. Muscle energy technique: definition, explanation, methods of procedure. J Am Osteopathic Assoc. 1981;81(4):249-54

26. Janda V. On the concept of postural muscles and posture in man. Aust J Physiotherapy. 1983;29(3):83-4. https://doi.org/10.1016/s00049514(14)60665-6.

27. Hinman RS, Hunt MA, Creaby MW, Wrigley TV, McManus FJ, Bennell KL. Hip muscle weakness in individuals with medial knee osteoarthritis. Arthritis Care Res. 2010;62(8):1190-3. https://doi.org/10.1002/acr.20199.

28. Bolgla LA, Malone TR, Umberger BR, Uhl TL. Hip strength and hip and knee kinematics during stair descent in females with and without patellofemoral pain syndrome. J Orthopaedic Sports Phys Therapy. 2008;38(1):12-8. https://doi.org/10.2519/jospt.2008.2462.

29. Tsai TY, Dimitriou D, Li G, Kwon YM. Does total hip arthroplasty restore native hip anatomy? Three-dimensional reconstruction analysis. Int Orthop. 2014;38(8):1577-83. https://doi.org/10.1007/s00264-014-2401-3.

\section{Publisher's Note}

Springer Nature remains neutral with regard to jurisdictional claims in published maps and institutional affiliations.

\section{Ready to submit your research? Choose BMC and benefit from:}

- fast, convenient online submission

- thorough peer review by experienced researchers in your field

- rapid publication on acceptance

- support for research data, including large and complex data types

- gold Open Access which fosters wider collaboration and increased citations

- maximum visibility for your research: over $100 \mathrm{M}$ website views per year

At $\mathrm{BMC}$, research is always in progress.

Learn more biomedcentral.com/submissions 